\title{
Optimal foraging in marine ecosystem models: selectivity, profitability and switching
}

\author{
André W. Visser ${ }^{1, *}$, Øyvind Fiksen ${ }^{2,3}$ \\ ${ }^{1}$ Centre for Ocean Life, National Institute for Aquatic Resources, Technical University of Denmark, Kavalergaard 6, \\ 2920 Charlottenlund, Denmark \\ ${ }^{2}$ Department of Biology, University of Bergen, 5020 Bergen, Norway \\ ${ }^{3}$ Uni Research, 5020 Bergen, Norway
}

\begin{abstract}
One of the most troubling aspects of ecosystem models is the use of rather arbitrary feeding and preference functions. The predictions of plankton functional type models have been shown to be highly sensitive to the choice of foraging model, particularly in multiple prey situations. Here we propose ecological mechanics and evolutionary logic as a solution to diet selection in ecosystem models. When a predator can consume a range of prey items, it has to choose which foraging mode to use, which prey to ignore and which ones to pursue, and animals are known to be particularly skilled in adapting their diets towards the most profitable prey items. We present a simple algorithm for plankton feeding on a size-spectrum of prey with particular energetic content, handling times and capture probabilities. We show that the optimal diet breadth changes with relative densities, but in a different way to the preference functions commonly used in models today. Indeed, depending on prey class resolution, optimal foraging can yield feeding rates that are considerably different from the 'switching functions' often applied in marine ecosystem models. Dietary inclusion is dictated by 2 optimality choices: (1) the diet breadth and (2) the actual feeding mode. The optimality model does not generate 'safety in low densities' for prey, as the 'switching function' does in ecosystem models, unless predators are shifting feeding mode adaptively. The actual diet, feeding rate and energy flux in ecosystem models can be determined by letting predators maximise energy intake or more properly, some measure of fitness where predation risk and cost are also included. An optimal foraging or fitness-maximising approach will give marine ecosystem models a sound principle to determine trophic interactions.
\end{abstract}

KEY WORDS: Grazing $\cdot$ Diet composition $\cdot$ Feeding mode $\cdot$ Fitness $\cdot$ Size selection

\section{INTRODUCTION}

The question of how to formulate behavioural or adaptive interactions between predators and prey in ecosystem- or food-web models has been around for a long time in the general ecological literature (Abrams 1984, Schmitz 2010, reviewed in Abrams 2010). More recently, the appearance of more ecologically resolved models of marine systems has generated a revived interest in the construct and parameterization of mixed-diet formulations in these systems as well
(Gentleman et al. 2003, Gentleman \& Neuheimer 2008, Anderson et al. 2010, Prowe et al. 2012a).

Marine ecosystem models have been strongly influenced by Fasham et al. (1990), and the use of parameterised preference functions has become the standard since then. The preferences can either be a fixed value or a function of relative abundances, often referred to as 'switching'. Different modelling options give different outcomes for the predicted diversity, structure and stability of the food web and ecosystem (Anderson et al. 2010, Prowe et al. 2012b). 
But how do we know which foraging function is the right one? Clearly, feeding functions that yield lower food intake for a predator when the abundance or availability of other prey increases are flawed (Gentleman et al. 2003). Here, we argue that the best diet model to use is one that maximises energy intake for the predator, based on general optimal foraging assumptions. We also point to algorithms where the predation risk involved in foraging is included in the choice of foraging mode and diet.

Animals have evolved a behavioural rationality in the economics and energetics of how they exploit their environment. This is the main justification for the optimality principle in behavioural ecology (Mangel \& Clark 1988), and it can be used to predict what animals do in trade-off situations (e.g. Krebs \& Kacelnik 1991). Optimality is also a sound principle to apply in ecosystem or food-web models (Smith et al. 2011) where organisms (or the modellers) face decisions about which organisms to include in their diet and which to ignore. According to classical optimal foraging theory (Charnov 1976), prey should be ignored if its energy value relative to the time it takes to consume the prey is low compared to alternative prey in the environment. By the same reasoning, a low probability of capturing a given prey type may also make it unprofitable if the time could be better spent searching for alternative prey. Optimal feeding on a mixture of prey types is often referred to as optimal diet breadth (ODB). This topic has gained much attention in simulations of complex systems in ecology (Petchey et al. 2008, Heckmann et al. 2012), where its role in ecosystem stability and biodiversity has been highlighted.

A further facet of optimal foraging in the plankton is the issue of foraging mode (e.g. ambush versus cruise feeding, habitat selection, etc.). Indeed, similar optimality reasoning may be applied to determine when zooplankton (e.g. a copepod) should switch between foraging modes when exposed to a mixed diet (e.g. phytoplankton and smaller zooplankton) (Visser et al. 2009, Mariani \& Visser 2010). Specifically, different foraging modes target different sections of the prey field, usually through the mechanics of the encounter process, and while the optimal diet for a given feeding mode can be estimated by the ODB model, the actual choice of feeding mode depends on the fitness afforded the grazer - a trade-off between benefit (ingestion), costs and risk. We show that the ODB model will not generate 'safety in low densities' for prey (i.e. reduced grazing pressure when alternate prey is more plentiful) as the 'switching function' does in ecosystem models, but that there may be a rescue in low densities if the predators are shifting feeding mode adaptively. The ODB and adaptive switch among foraging modes could become an integral part of current ecosystem models, and replace some of the loosely founded 'preference functions'. Our main goal here is to show how this can be done in a size-structured ecosystem model using an optimality assumption about both diet inclusion and foraging mode.

\section{FUNCTIONAL RESPONSE MODELS}

We contend that whatever the specific formulations employed in models, they should be based on mechanisms that are ecologically meaningful. To this end we assert that Holling type II functional response ('Holling's disk equation'; Holling 1959) should be the primary structure of all trophic interactions. We make this assertion in the light that Holling II is inherently individual-based, wherein traits of predators and prey can be incorporated in a transparent manner. It is also explicitly mechanistic. It is based on measurable subprocesses (encounter rates and handling times) and can be readily tailored to include changing environmental conditions (e.g. light, turbulence, predator and prey abundances) or shifts in behaviour (e.g. foraging effort, feeding mode, defensive strategies). With regard to the latter, it is particularly amenable to include adaptive behaviour, wherein the predator and prey jockey respectively for their best foraging and defence strategies in the fitness stakes.

Holling I (with saturation) is a variant of type II and can be derived from essentially the same principles where the predator has the ability to process $>1$ prey item at a time (Sjöberg 1980, Wirtz 2012a). The Ivlev type functional response falls somewhere between a Holling type I and II with a specific processing capacity (Wirtz 2012a). In contrast, a Holling type III functional response involves some behavioural response of the predator to the prey field, either through a habitat shift (e.g. Murdoch et al. 1975) or learning (Real 1977) or from a shift in the predator's foraging mode (e.g. cruising versus ambush; Kiørboe et al. 1996, Gismervik \& Andersen 1997). That is, a Holling type III is an emergent functional response dictated by encounter rates, processing time and the adaptive fitness-seeking behaviour of grazers. It should not be prescribed a priori in model applications; rather, the underlying mechanisms from which it arises should be explicitly modelled to insure some fidelity in the ensuing trophic dynamics. 
Finally, a popular class of functional response formulations follow a Michaelis-Menten or Monod description. While it is true that these descriptions are equivalent to a Holling type II for a single prey type, their interpretation becomes clouded when multiple prey types are considered. Specifically, encounter rates and handling times can be readily classified for different prey types, but what is the half saturation concentration $(K)$ for a prey mixture? It is not constant but varies with the relative proportion of various prey types in the diet (Prowe et al. 2012a). Many (if not most) modellers use the estimate of $K$ from Hansen et al. (1997)-despite large scatter (orders of magnitude) and unclear meaning-concluding that the half saturation in terms of biomass is the same across 8 orders of magnitude of predator mass. To avoid this confounding effect, we suggest that Michaelis-Menten or Monod descriptions of functional responses for mixed diets be abandoned in favour of equivalent but more transparent and mechanistic Holling type II descriptions.

\section{MODELS}

\section{Optimal diet breadth and profitability}

The concept of prey profitability (Charnov 1976) becomes evident when considering the optimal decision a predator should make when confronted by a newly encountered prey - whether it should include it in its diet or not. In its original form, the argument is that if $g$ is the mean ingestion rate, $e_{i}$ is the cell content (e.g. in terms of carbon or energy) of the newly encountered prey type $i$, and $h_{i}$ is the time needed for the predator to handle it, then the criterion for inclusion is $e_{i} / h_{i}>g$, while if $e_{i} / h_{i}<g$ then the prey item should be rejected (see Table 1 for definitions, dimensions and units). This is the diet that maximises food intake rate.

Table 1. Glossary of terms

\begin{tabular}{|c|c|c|}
\hline Symbol & Description & Units \\
\hline$a_{i}$ & Prey abundance for prey class $i$ & $\mathrm{~m}^{-3}$ \\
\hline$\beta$ & Encounter kernel (maximum clearance rate) & $\mathrm{m}^{3} \mathrm{~s}^{-1}$ \\
\hline$\beta_{i}$ & Encounter kernel for prey class $i$ & $\mathrm{~m}^{3} \mathrm{~s}^{-1}$ \\
\hline$\beta_{i, m}$ & Encounter kernel for prey class $i$ and feeding mode $m$ & $\mathrm{~m}^{3} \mathrm{~s}^{-1}$ \\
\hline$C_{m}$ & Cost for feeding mode $m$ & $\mathrm{gC} \mathrm{s}^{-1}$ \\
\hline$C_{\mathrm{TOT}}$ & Total biomass concentration & $\mathrm{gC} \mathrm{m}^{-3}$ \\
\hline$e_{i}$ & Prey carbon content for prey class $i$ & $\mathrm{gC}$ \\
\hline$e(r)$ & Prey carbon content as a function of cell size & $\mathrm{gC}$ \\
\hline$\varepsilon_{i, m}$ & Escape probability of prey type $i$ when exposed to feeding mode $m$ & Dimensionless \\
\hline$f_{m}$ & Fitness for feeding mode $m$ & $\mathrm{gC}$ \\
\hline$g$ & Grazing rate & $\mathrm{gC} \mathrm{s}^{-1}$ \\
\hline$g_{k}$ & Grazing rate for all prey class $i \leq k$ & $\mathrm{gC} \mathrm{s}^{-1}$ \\
\hline$g_{\max , m}$ & Maximum grazing rate for feeding mode $m$ & $\mathrm{gC} \mathrm{s}^{-1}$ \\
\hline$g_{\Theta 0}$ & Grazing rate for non-switching preference function; $\theta_{i}=\phi_{i}$ & $\mathrm{gC} \mathrm{s}^{-1}$ \\
\hline$g_{\Theta 1}$ & Grazing rate for switching preference function; $\theta_{i}=\phi_{i}$ & $\mathrm{gC} \mathrm{s}^{-1}$ \\
\hline$h_{i}$ & $\begin{array}{l}\text { Handling time for prey class } i \text {. This can be subdivided into time accruing to all attacks ( } h_{i a i} \\
\text { pursuit, capture, etc.) and time devoted to processing captured prey }\left(h_{i d} \text {, ingestion, digestion, etc.) }\right.\end{array}$ & S \\
\hline$h(r)$ & Handling time as a function of cell size & $\mathrm{s}$ \\
\hline$i$ & Profitability index, arranged in descending order so that $p_{i} \geq p_{i+1}$ & Dimensionless \\
\hline$\mu_{0}$ & Baseline mortality rate, independent of feeding mode & $\mathrm{s}^{-1}$ \\
\hline$\mu_{m}$ & Additional mortality rate associated with feeding mode $m$ & $\mathrm{~s}^{-1}$ \\
\hline$n(r)$ & Cell size spectrum giving the abundance of cells $\left(\mathrm{m}^{-3}\right)$ per unit size $(\mathrm{m})$ & $\mathrm{m}^{-4}$ \\
\hline$N_{\text {TOT }}$ & Scaling factor for cell size spectrum & $\mathrm{m}^{-4}$ \\
\hline$p_{i}$ & Profitability for prey class $i$ & $\mathrm{gC} \mathrm{s}^{-1}$ \\
\hline$\phi_{i}$ & Catchability for prey class $i$ & Dimensionless \\
\hline$\phi(r)$ & Catchability as a function of cell size & Dimensionless \\
\hline$r$ & Size (equivalent spherical radius) of cells & $\mathrm{m}$ \\
\hline$R$ & Size (radius) of grazer & $\mathrm{m}$ \\
\hline$\theta_{i}$ & Innate availability for prey class $i$ & Dimensionless \\
\hline$\Theta_{i}$ & Preference function for prey class $i$ & Dimensionless \\
\hline$u_{m}$ & Grazer swimming speed in feeding mode $m$ (e.g. $u_{m}=0$ for ambush feeding mode) & $\mathrm{m} \mathrm{s}^{-1}$ \\
\hline$V_{i}$ & Swimming speed for prey type $i$ (e.g. $v_{i}=0$ for non-motile prey) & $\mathrm{m} \mathrm{s}^{-1}$ \\
\hline
\end{tabular}


To this we add the concept of catchability, assuming that an attack on a prey will not always lead to a successful capture. If the probability of an attack being successful is $\phi_{i}$, then the expected value of return on an attack is $\phi_{i} e_{i}$. We define prey profitability as:

$$
p_{i}=\phi_{i} \frac{e_{i}}{h_{i}}
$$

The optimal diet of the predator should include all prey items with profitability above some critical value, and exclude less profitable ones. The critical profitability value is equal to the grazing rate attained by including all available prey items of superior profitability. This can be shown graphically by arranging prey according to profitability $p_{i}$ (Fig. 1). There is a prey profitability where including it in the diet will lower the ingestion rate. Specifically, writing $a_{i}$ as the abundance of prey within each class, and $\beta_{i}$ as the encounter kernel (search rate) of the grazer with prey in size class $i$, and ordering prey classes such that $p_{i}>p_{i+1}$, then the optimal grazing rate is:

$$
g_{\max }=g_{k}=\frac{\sum_{i=1}^{k} \phi_{i} e_{i} a_{i} \beta_{i}}{1+\sum_{i=1}^{k} h_{i} a_{i} \beta_{i}}
$$

where $k$ is such that $g_{k}$ is a maximum, a condition ensured by $g_{k}>p_{j}$ for $j>k$. We will provide a more concrete example in the following section 'Sizebased application'.

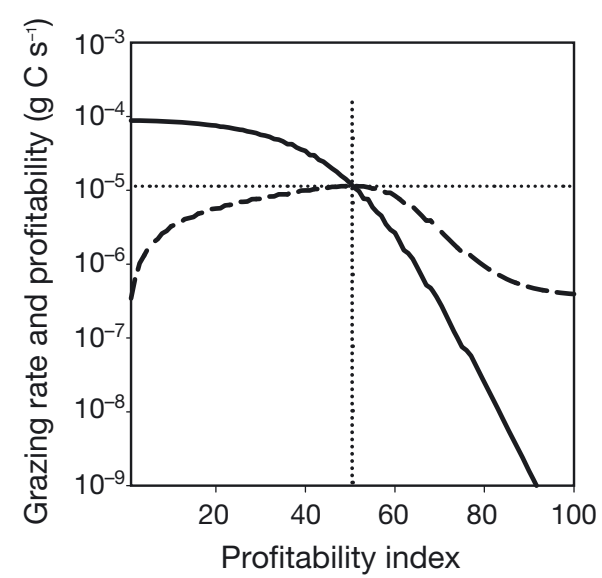

Fig. 1. Relationship between prey profitability (solid line) and optimal grazing rate $g$ (dashed line), both of which are ranked according to descending profitability $p$. The point where the 2 lines cross defines the critical profitability index $k$ (vertical dotted line) and critical profitability $p_{k}$. It is also the point where $g$ attains its maximum value $g_{\max }$ (horizontal dotted line). That is $g_{\max }=p_{k}$. Including prey with $p<p_{k}$ (i.e. with profitability index $>k$ ) will invariably reduce $g<g_{\max }$
In this formulation we assume that handling time accrues to all attacks (i.e. pursuit and capture). In order to include handling times related to processing captured prey (i.e. ingestion and digestion), one can introduce a slightly different formulation: $h_{i}=h_{i a}+$ $\phi_{i} h_{i d}$, where $h_{i d}$ is the time to pursue and capture a prey and $h_{i d}$ is the time it takes to ingest and digest it. While we do not specifically pursue this here, we note that the distinction between capture and processing times should be given due attention in practical applications.

While the decision to include a prey or not does not specifically depend on the abundance of that prey type, the grazing rate to which profitability is compared and the ensuing inclusion criterion does depend on the abundance of all included prey types. An intuitive result from this model is that large prey will be ignored as they become difficult to catch, while easy-to-catch but small prey will be ignored as their energy content becomes too low to warrant the time it takes to catch them, leading to a preferred size range. Note however that the breadth of this preferred range is expected to be dynamic, changing its width in accordance with the abundance of prey types available to the grazer. This algorithm can be extended to also include a continuous (every time step) assessment of all alternative foraging modes (one ODB for each) and then choose the best one-a well-informed predator using simple fitness-seeking heuristics alternating among foraging modes and diets.

\section{Size-based application}

While the general concept of prey selectivity in terms of profitability can be applied to any number of cases, we wish to highlight its role in determining a grazer's size preference and size class breadth of diet (Hansen et al. 1997, Fuchs \& Franks 2010, Wirtz 2012b). Size after all is the key trait governing trophic interactions, particularly in determining the carbon and energy content of a prey item. Further, it can be supposed to influence both handling time and catchability and so structure the profitability of a predator's diet. We envisage a predator (e.g. adult copepod) of size $R$ (equivalent spherical radius) feeding on a range of prey of various size classes $r$. We assume that both handling time and catchability depend on the size of the prey relative to that of the predator, and the general size-dependent carbon content, handling times and catchability are given by: 


$$
\begin{gathered}
e(r)=c_{0} r^{n_{e}} \\
h(r)=h_{0}+h_{1}\left(\frac{r}{R}\right)^{n_{h}} \\
\phi(r)=\left(1-\frac{r}{R}\right)^{n_{p}}
\end{gathered}
$$

The exponents $n_{e}, n_{h}$ and $n_{p}$ control the shape of these functions, and illustrative examples together with the resulting profitability function are shown in Fig. 2. For particular applications, these functions should reflect the groups of predators and prey in question, and while handling times and catchability may be difficult to specify, it is certainly possible to estimate or model them for specific groups of organisms (Paffenhöfer 1984, Wirtz 2012b). In fact, these processes depend on real traits of prey organisms (e.g. spines or exoskeletons), the effect of these on handling time (both capture and digestion times), catchability, and even energy content, and provide a mechanistic link to the survival benefits the traits confer on prey organisms in the framework suggested here.
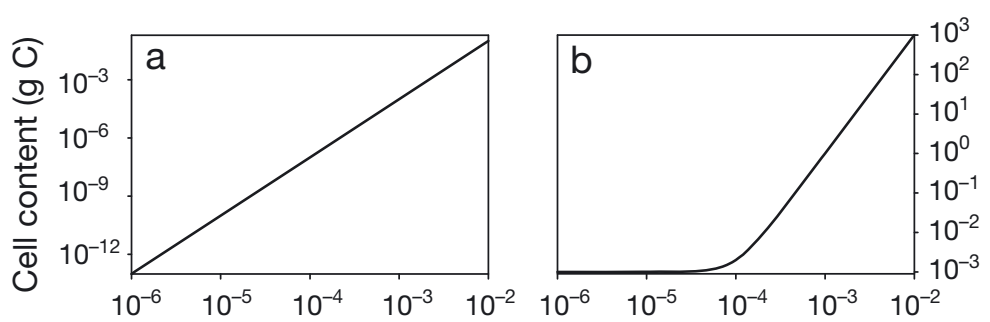

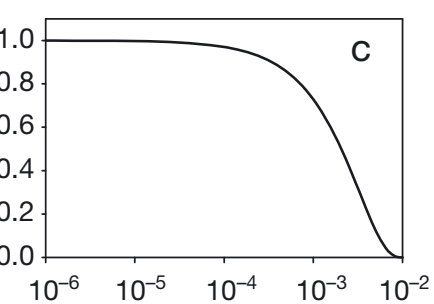

Cell radius $(\mathrm{m})$

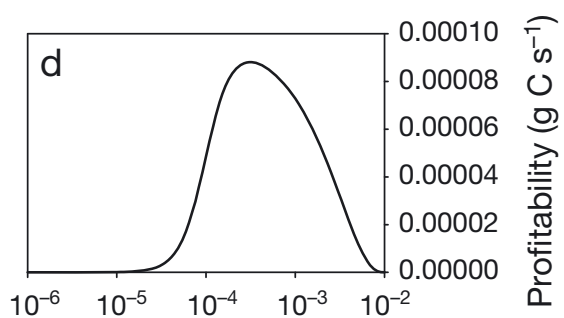

Cell radius $(\mathrm{m})$
Fig. 2. (a) Cell content is estimated as being proportional to cell volume $\left(n_{e}=3\right)$ so that $c_{0}=10^{5} \mathrm{gC} \mathrm{m}^{-3}$ is the cell carbon:volume ratio (Hansen et al. 1997). (b) For handling time, we assume there is a minimum handling time per prey item, $h_{0} \approx 1 \mathrm{~ms}$, while for larger particles, handling time increases cubically $\left(n_{h}=3\right)$ with prey:predator ratio to a maximum of $h_{1}=1000 \mathrm{~s}$ at a predator:prey ratio of 1. Maximum handling time is somewhat greater than that associated with copepods capturing large food items (e.g. order $100 \mathrm{~s}$; Williamson 1980), but is consistent with digestion and gut passage times for large prey (800 to 1200 s; Dutz et al. 2008). (c) Following Petchey et al. (2008), catchability reflects the general trend that prey much smaller than the predator are easily caught $(\phi \rightarrow 1$ for $r / R<<1)$, while prey of size approaching the predator are more difficult to catch ( $\phi \rightarrow 0$ as $r / R \rightarrow 1$ ). Parameter setting is $n_{p}=3$ (Eq. 3). (d) The resulting profitability shows the profitability of prey of a given size combining the effects of energy content, handling time and capture probability

$$
n(r)=N_{\mathrm{TOT}}\left(\frac{r}{r^{*}+r}\right)^{n_{u}}\left(\frac{r^{*}}{r^{*}+r}\right)^{n_{d}}
$$

defined over the size range $r \in\left[r_{0}, r_{1}\right]$, and expressed in particles number density per length category, $\mathrm{m}^{-3}$ $\mathrm{m}^{-1}$. When $n_{u}$ and $n_{d}$ are both positive, this gives a distribution with a peak centred on $r^{*}$. The total biomass concentration in the system $C_{\mathrm{TOT}}$ is thus given by:

$$
C_{\mathrm{TOT}}=\int_{r_{0}}^{r_{1}} e(r) \cdot n(r) \cdot \mathrm{d} r
$$

We can thus set $C_{\text {TOT }}$ to environmentally relevant values, from which the scaling factor $N_{\text {TOT }}$ and thus quantified particle size spectra are determined. Fig. 3 shows an example, and is qualitatively similar to In choosing this example, we are mindful that in a full size-based ecosystem model (e.g. Fuchs \& Franks 2010), the actual size spectrum will be dynamic, being governed by the trophic interactions of growth and mortality within and between prey size classes.

Provided we choose the width of each size class to be small (i.e. in general this means a large number of size classes), then the integration in Eq. (7) can be approximated by the sum:

$$
C_{\mathrm{TOT}}=\sum_{i_{\min }}^{i_{\max }} e_{i} \cdot a_{i}
$$

where $a_{i}=n_{i} \delta_{i}$ is the abundance of particles in the size class $i_{1}$ and $\delta_{i}$ is the width of the size class.

\section{Preference and switching functions in recent marine ecosystem models}

Preference functions generally proceed from the concept of innate availability $\theta_{i}$ (Stock et al. 2008) indicating the proportion of prey in a particular class that is available to a predator. Dietary preference based on innate availability is termed passive selection (Gentleman et al. 2003). The underlying concept here is that the predator can only perceive and/or capture a 
limited range of prey due to some physical restrictions in its foraging mechanisms (e.g. inability to handle extremely large or extremely small prey, conflicts between feeding modes and encounter rates on certain prey classes). While this can be seen as being related to the catchability $\phi_{i}$ we have introduced above, it is not identical. Preference functions of the passive selection kind are generally prescribed as being strongly size-dependent, reflecting general trends of dietary inclusion found in nature (e.g. Cohen et al. 1993). For example, Banas (2011) introduces a preference function that is a log-Gaussian distribution, centred about one order of magnitude smaller that the predator.

Marine-food web models often also include a factor indicating a preference for prey classes that dominate the prey community in terms of biomass. This is generally referred to as switching (Murdoch et al. 1975) or active selection (Gentleman \& Neuheimer 2008), in that it reflects behavioural changes in predator feeding modes that actively target certain prey classes the underlying assumption being that it is best for the predator to target those prey classes that are most abundant. This, together with innate availability leads to a general preference function of the form:

$$
\Theta_{i}=\theta_{i}\left(\theta_{i}^{n_{s}-1}\left(e_{i} a_{i}\right)^{n_{s}} / \sum_{i}\left(\theta_{i} e_{i} a_{i}\right)^{n_{s}}\right)^{m_{s}}
$$
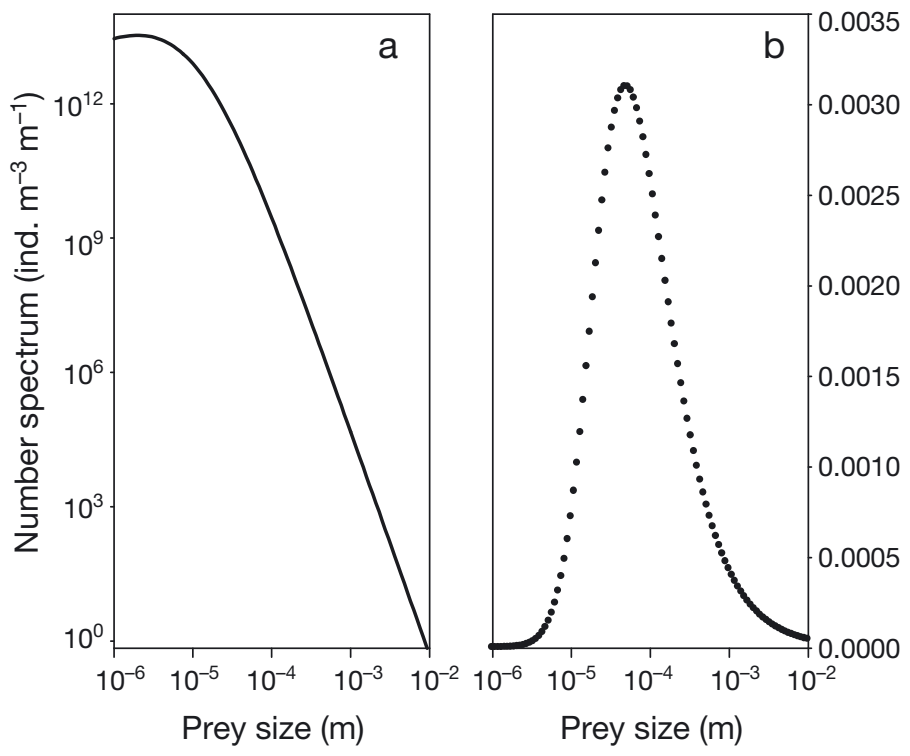

Fig. 3. (a) Number density spectrum $n(r)$ following from Eq. (6), and (b) the associated biomass density per size class $e_{i} a_{i}$ used in the illustrative example. In the latter, we used 100 size bins of logarithmically increasing width. Parameter setting: $n_{u}=1, n_{d}=5, r^{*}=10 \mu \mathrm{m}$. Total biomass in the system is set at $C_{\mathrm{TOT}}=\sum e_{i} a_{i}=0.1 \mathrm{gC} \mathrm{m}^{-3}$
(Stock et al. 2008). The exponents $n_{s}$ and $m_{s}$ can be varied to give a stronger or weaker switching mechanism. While it has the same functionality, this formulation is slightly different from that given in Stock et al. (2008), so that for $n_{s}=m_{s}=1$, the preference function of Fasham et al. (1990) is returned, while $m_{s}$ $=0$ returns a preference function without active selection (e.g. Banas 2011).

The ensuing grazing rate in existing models is generally formulated as:

$$
g_{\Theta}=\frac{\sum_{i=1}^{i_{\max }} \Theta_{i} e_{i} a_{i} \beta_{i}}{1+\sum_{i=1}^{i_{\max }} \Theta_{i} h_{i} a_{i} \beta_{i}}
$$

Note how the preference function enters this formulation; it does so as a pre-multiplier to the encounter rate. That is, the predator essentially ignores a proportion of the prey encountered in accordance with its preference. This is in contrast to our catchability formulation (Eq. 2), which does not automatically predicate that prey of low catchability should be ignored.

\section{Optimal diet breadth}

Using the size-profitability relationships together with an illustrative size spectrum, we can now calculate ODB for a range of total biomass concentrations. Without going into specific details of the feeding mode employed by the predator (this will be discussed in 'Results and discussion' section 'Comparing predictions for optimal foraging to prescribed preference and switching functions'), we choose a constant specific maximum search rate (i.e. volume searched for prey per body volume per unit time) of $\beta / V=$ $10^{6} \mathrm{~d}^{-1}$, where $V$ is the predator's body volume. This appears to be a relatively robust relationship - albeit with considerable variance-across a wide range of marine species (Kiørboe 2011). There is also a considerable range in the search rate among predators; they may swim faster if prey are scarce, for instance (Follows \& Dutkiewicz 2011). This requires more computations, but can readily be included, although we leave that element out here. Similarly, the issue of digestion limitation and how gut constraints influence ODB is left out, since this would require a second state variable in addition to organism size. In general, digestion limitation will increase the benefits of adaptive prey selection if prey differ in quality. 


\section{RESULTS AND DISCUSSION}

\section{Should zooplankton be selective in their diet?}

For a large adult copepod $\left(R=10^{-2} \mathrm{~m}\right)$, we calculate ODB using the size-based profitability schedule illustrated in Fig. 2d. We do this keeping the shape of the size spectrum constant, but vary the total biomass of prey. The maximum size of prey included in the diet is slightly less than the size of the predator (Fig. 4a), reflecting the high profitability of large prey despite low catchability and long handling times (Fig. 2d). The diet breadth initially spans the entire size spectrum up to about $C_{\mathrm{TOT}}=10^{-5} \mathrm{gC} \mathrm{m}^{-3}$, after which an increasing portion of prey items from the small end of the size spectrum (i.e. low profitability) become excluded from the diet. From about $C_{\mathrm{TOT}}=10^{-2} \mathrm{gC}$ $\mathrm{m}^{-3}$, prey from large size classes also become excluded, reflecting increased handling times and decreased catchibility. That is, there is an overall narrowing of ODB with increased total prey biomass.
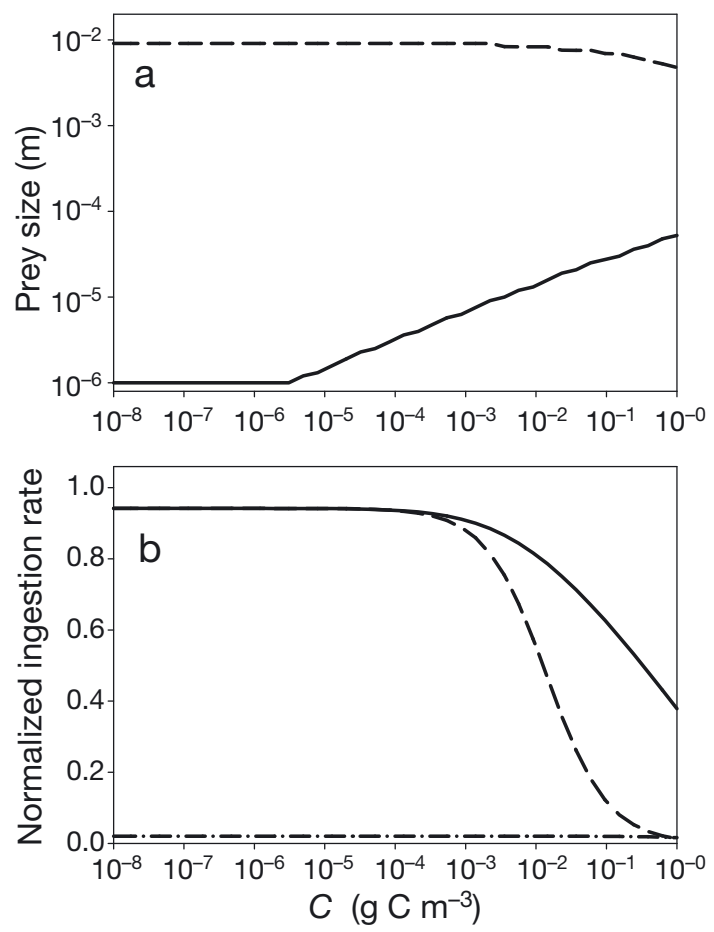

Fig. 4. (a) Optimal diet breadth as delimited by the minimum $r_{\min }$ (solid line) and maximum inclusive size $r_{\max }$ (dashed line), and (b) normalised ingestion rate for a $1 \mathrm{~cm}$ predator feeding on a prey size spectrum (shape according to Fig. 3) for variable total prey biomass ranging from $10^{-8}$ to $1 \mathrm{gC} \mathrm{m}^{-3}$ $\left(=\mathrm{mgC}^{-1}\right)$. In $(\mathrm{b})$, variously determined grazing rates are $g_{\max }$ (solid line) based on profitability, preference with no switching $g_{\ominus 0}$ (dashed line) and preference with switching $g_{\Theta 1}$ (dot-dashed line)
This change in ODB is moderate; it still spans $>2$ orders of magnitude in prey size even at extremely high total carbon concentrations and is not particularly sensitive to the shape of the prey size spectrum. This is consistent with general observations that the ODB of copepods span nearly 3 orders of magnitude (Fuchs \& Franks 2010), and are food-limited with half saturation constants of the order of $200 \mathrm{mgC} \mathrm{m}^{-3}$ (Hansen et al. 1997). That is, copepods live in a dilute environment (Kiørboe 2011) and simply cannot afford to be too selective; they should consume most of what they encounter. In the vocabulary of optimal foraging theory, they are search-limited and not handling-limited.

\section{Comparing predictions for optimal foraging to prescribed preference and switching functions}

This gives us 3 different estimates of ingestion rates: (1) $g_{\max }$ as per Eq. (2); (2) $g_{\ominus 0}$ as per Eq. (10) assuming no 'switching' $\left(m_{s}=0\right.$, only innate availability); and (3) $g_{\ominus 1}$ as per Eq. (10) including 'switching' that is kept at minimal complexity, with $n_{s}=m_{s}=$ 1 (i.e. both 'switching' and innate availability). To make an internally consistent comparison, we use the same function (Eq. 5) to describe both catchability $(\phi)$ and innate availability $(\theta)$ in both models. The rationale here is that whatever other factors may be involved, we can say with some certainty that $\phi>\theta$, so that setting $\phi=\theta$ gives the most generous estimates of grazing $\left(g_{\ominus 0}\right.$ and $\left.g_{\ominus 1}\right)$ using the prescribed preference approach. We normalise all grazing rate estimates with respect to $\beta C_{\text {TOт }}$ (Fig. $4 \mathrm{~b}$ ).

As might be expected, grazing rate $g_{\max }$ determined by prey profitability gives the highest estimated energy intake across all total biomass concentrations. The innate availability estimate $g_{\ominus 0}$ follows closely at low concentrations but diverges to lower estimates at high concentrations. The estimate with switching, $g_{\Theta 1}$, is consistently lower by an order of magnitude.

The result that $g_{\max }>g_{\Theta 0}$ reflects the mechanism that for a prescribed preference function, the predators waste valuable time handling small prey with low energy content. This is an effect that becomes more pronounced at high total biomass concentrations when profitability optimisation dictates the exclusion of a growing fraction of small prey classes (Fig. 4a). In general, a prescribed preference function can at times give similar grazing rates as optimalityderived grazing, but will always give suboptimal rates at other times. A broad preference function will give suboptimal performance at high prey concen- 
trations as time is wasted on handling low-energycontent prey, while a narrow preference function will be suboptimal for low prey concentrations where potentially valuable are excluded from the diet.

That the switching estimate $g_{\Theta 1}$ is less than the optimal grazing rate $g_{\text {max }}$ is at first not surprising, but why is it so much less? It turns out that this is symptomatic of a systematic failure of the commonly applied switching formulation (from Fasham et al. 1990) and variants thereof (e.g. Stock et al. 2008). Specifically, using abundance-weighted preference functions leads to a dependence of estimated grazing rates on the number of prey classes included in a model. To illustrate this, consider the simple thought experiment presented in Fig. 5. We consider 2 models of exactly the same system, one where there are 2 prey classes A and $B$, and the other where we arbitrarily subdivide prey class A into 2 subclasses $A_{1}$ and $A_{2}$. The first consequence of this is that the most preferred prey item shifts from A in Model 1 to B in Model 2. More problematic is that the predicted total grazing rate of the predator is consistently lower for Model 2 than for Model 1, and all because we chose an arbitrary subdivision of the prey classes in our model. Exactly the same problem crops up whether the weighting functions are constructed in terms of abundance, clearance rate or ingestion rates, and it becomes more pronounced as the prey field is subdivided into more and more classes. It arises essentially from the nonlinearity of the functional response-Holling type III for instance-where the sum of averages is not the same

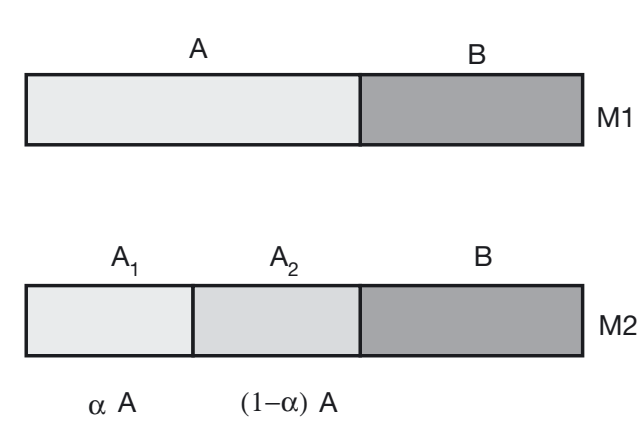

Fig. 5. Two model constructions, M1 and M2 for the same system, the difference being that in $\mathrm{M} 2$, the prey class A is subdivided according to a partitioning function $\alpha$. All other parameters are equivalent, and the models are identical when $\alpha=0$ or 1 . Grazing rate is calculated according to a 'switching function' where prey preference is a function of prey abundance. In this context, the choice of $\alpha$ has no ecological significance and yet the model predicts different relative grazing pressure per prey class (a): B (solid), $\mathrm{A}_{1}$ (dotdash) and $A_{2}$ (dash), as well as total grazing rate (b) of model M2 compared to M1 as a function of $\alpha$ as the averaged sum. These effects can of course be calibrated out in specific model applications, and most published models use subdivisions that are not arbitrary. However, there is something very unsettling in the idea that model architecture can have such an effect on seemingly fundamental ecological processes.

This underscores an important concept for both modellers and ecologists. There are demonstrable density-dependent effects in nature (e.g. Murdoch et al. 1975, Gismervik \& Andersen 1997), but the density of what exactly? Is it the density of conspecifics, similar functional types, members of the same or some alternate targeted prey group? We have no immediate answer to this, but in what follows, we make a suggestion as to how this may be addressed for mixed diets in marine ecosystems.

\section{Switching and optimal foraging mode}

We now turn to the question of how switching can be incorporated in grazing rates according to our optimality criterion. Again, we wish to derive this in a way that is as mechanistic and evolutionarily consistent as possible. Our premise is that realised switching on various prey types is an emergent property of the predator switching between various feeding modes, and it is the feeding modes that dictate dietary inclusion.

Copepods for instance can in general choose between 3 different feeding modes (Kiørboe 2011):
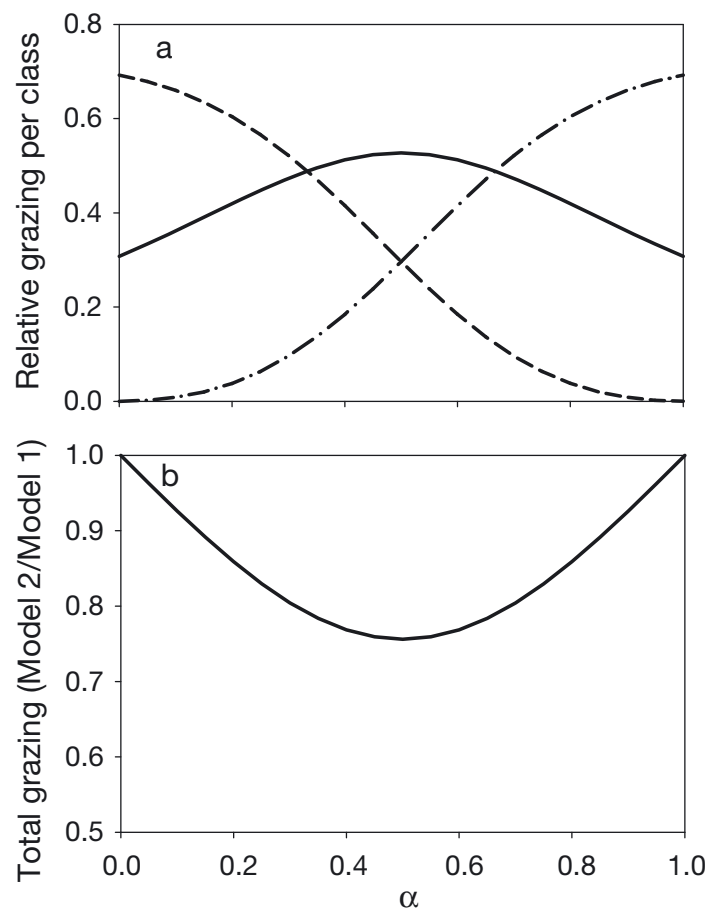
suspension (scanning-current) feeding, cruise feeding and ambush predation. Each of these preferentially selects for certain prey types predicated primarily on prey motility. For instance, ambush feeding selects for motile prey but is pretty much useless in finding immotile prey. On the other hand, a suspension feeding current, while good for capturing immotile prey, may alert motile prey to predation risk and so elicit an escape reaction. Furthermore, different feeding modes also incur greater or lesser energetic costs, as well as exposing the predator to different predation risks of its own. Ambush predation will have low energetic costs and low risk, while both will be higher for suspension feeding and higher still for cruise feeding. Thus optimality of feeding mode is not simply dictated by the maximisation of ingestion rate, but should also reflect the inherent trade-offs it poses against costs and risks.

Predicated on the same principle of profitability put forward in section 'Optimal diet breadth and profitability', the maximum ingestion rate for each feeding mode can be calculated as:

$$
g_{\text {max }, m}=g_{k, m}=\frac{\sum_{i=1}^{k} \phi_{i} e_{i} a_{i} \beta_{i, m}}{1+\sum_{i=1}^{k} h_{i} a_{i} \beta_{i, m}}
$$

where the subscript $m$ indicates an estimate for a specific feeding mode. The feeding mode will have no direct effect on prey profitability, although in general, motile prey will have lower catchability and higher handling time than non-motile prey and thus lower profitability in general (food quality notwithstanding). Neither will it affect abundance. It will however, affect the encounter kernels $\beta_{i, m}$. For instance, a simple kinetic encounter kernel formulation may be:

$$
\beta_{i, m}=\sigma_{i, m} \cdot\left(1-\varepsilon_{i, m}\right) \cdot\left(u_{m}^{2}+v_{i}^{2}\right)^{1 / 2}
$$

where $v_{i}$ is the speed of the prey, $u_{m}$ is the speed of the predator relative to the fluid, $\sigma_{i, m}$ is the perception cross-sectional area of the predator in mode $m$ on prey type $i$, and $\varepsilon_{i, m}$ is the probability that the prey will make a successful escape before it can be perceived by the predator in mode $m$. For copepod feeding modes, a possible set of interaction rules could be posed as follows. For ambush feeding $\left(u_{m}=0\right), \varepsilon_{i, m} \approx 0$ for all prey types, while for scanning and cruise feeding $\left(u_{m}>0\right), \varepsilon_{i, m} \approx 0$ for non-motile prey and $\varepsilon_{i, m} \approx 1$ for motile prey. These rules would reproduce the motility-feeding mode interactions alluded to in the previous paragraph.

Note that there is a distinction between the effect of escape and catchability which has to do with whether the predator perceives the prey before it escapes or not. If it escapes before, then the effect is on the innate availability of that prey type; the predator simply doesn't see it so it does not figure in its profitability schedule. If it escapes after, then it enters into catchability and profitability, presumably with a lower value due to both lowered expectation of successful capture, and possibly also a higher handling time to account for pursuit.

Maximum ingestion is not the sole ingredient in determining feeding mode optimality - it includes a trade-off between benefits, risks and costs. We make this explicit in the formulation:

$$
f_{m}=\frac{g_{\text {max }, m}-c_{m}}{\mu_{0}+\mu_{m}}
$$

where $f_{m}$ is a measure of fitness, $C_{m}$ is cost (in terms of carbon loss rate), and $\mu_{m}$ is the marginal increase in mortality associated with feeding mode $m$. The assumption here is that cost and risk are functions of feeding mode, and not dependent on the prey encounter process. Essentially, Eq. (13) expresses the instantaneous contribution to the predator's reproductive value, and in the absence of any other expectations, should be optimised (Gilliam \& Fraser 1987, Visser 2007). The optimal feeding mode $m^{*}$ is thus that for which $f_{m^{*}}$ is maximised, and the optimal diet is that dictated by profitability and the optimal feeding mode.

Finally, we offer a fitness-weighted grazing estimate:

$$
g^{*}=\frac{\sum_{m}\left(f_{m} g_{\text {max }, m}\right)}{\sum_{m} f_{m}}
$$

where each feeding mode and subsequent grazing is proportionally enacted according to their relative fitness. The concept here is that grazers continually explore their fitness landscape, sacrificing absolute optimality in exchange for information. This provides a simple myopic heuristic that can be implemented in ecosystem models to mimic adaptive, risk-sensitive foragers. More time is spent in feeding modes imparting high fitness and less time in lower fitness feeding modes. A formulation along these lines would reintroduce some of the features of 'switching' functions without incurring the prey-type partitioning problem illustrated in Fig. 5. The ingestion term in Eq. (13) can be elaborated to include a more complete bioenergetic model, with a gut state variable, and where ingestion can more properly be replaced by growth. If growth satiates at increasing prey density, this algorithm will lead grazers to shift to less efficient foraging modes with lower $c_{m}$ and $\mu_{\mathrm{m}}$, and 
an extended benefit of higher food availability through reduced mortality from behavioural change (see Fiksen \& Jørgensen 2011).

This approach, either through fitness maximisation or a fitness-weighted estimate (Eq. 14), could result in switching where predators switch from one prey type (e.g. phytoplankton) to another (e.g. microzooplankton) as a result of relative abundances. This switching would bring about something akin to a refuge at low numbers, but only insofar as it extends to all prey with a similar susceptibility to a given feeding mode. As a proof of concept, we include model results for a predator feeding in either ambush or cruise mode, on a mixture of motile and non-motile prey of different size classes (Fig. 6). We also supply the MATLAB code for these calculations as supplementary material (www.int-res.com/articles/suppl/ m473p091_supp/). In this scenario, we assign both an added cost and an increased mortality risk to cruise feeding over ambush feeding. These are set as representative for an adult copepod (e.g. Acartia sp.) in a typical predator field (Visser 2007). There is an abrupt transition from cruise to ambush mode at a critical value of the ratio of motile to non-motile prey which is associated with a change in the ingestion

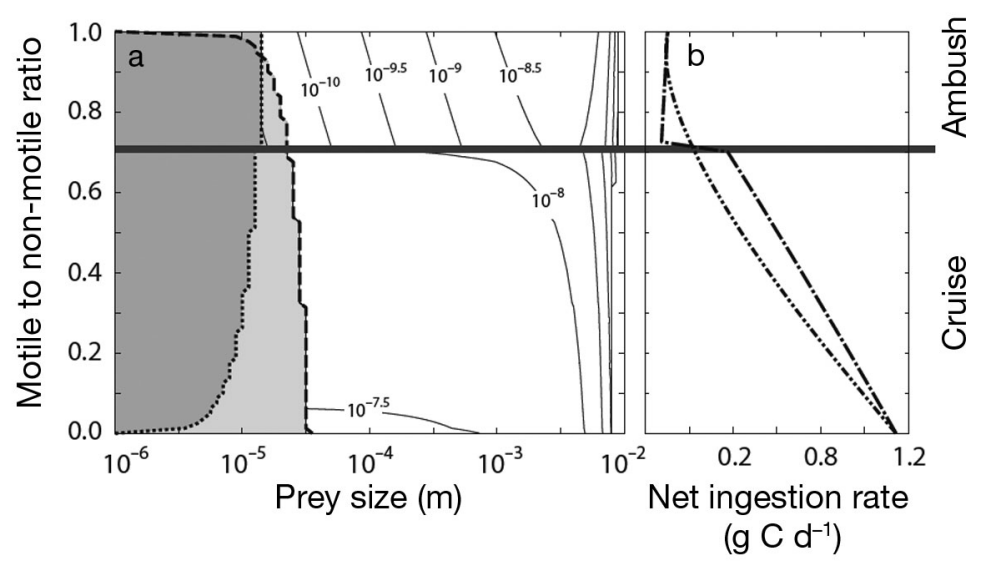

Fig. 6. Implication of fitness-regulated feeding mode switching (Eq. 13) on (a) grazing pressure $\left(\mathrm{gC} \mathrm{s}^{-1}\right)$ per size class and (b) the net ingestion rate $\left(\mathrm{gC} \mathrm{d}^{-1}\right)$ for fitness maximisation (dot-dash) and fitness-weighted (Eq. 14; dot-dot-dash) estimates respectively, for varying mixtures of motile and non-motile prey. The dotted and dashed lines to the left of panel (a) indicate the profitability size cut off for non-motile and motile prey respectively. In this example, we assume exclusive mode-dependent feeding (i.e. cruise on non-motile prey only and ambush on motile prey only), although this exclusivity can be readily relaxed (cf. supplement available at www.int-res.com/articles/suppl/m473p091_supp/). The size spectrum for both prey motility types is linear with equal biomass per logarithmic division, and the total prey biomass in the system is kept constant at $100 \mathrm{mgC} \mathrm{l}^{-1}$. Cruise feeding is given a cost penalty of $10^{-10} \mathrm{gC} \mathrm{s}^{-1}$ compared to ambush, as well as a 4 -fold increase in predation risk. Based on these fitness considerations, there is a transition from cruise to ambush when the motile to non-motile ratio is about $70 \%$ rate of the grazer. For the prey field, grazing pressure shifts not only from non-motile to motile prey, but

Although beyond the scope of this present work, profitability and fitness considerations would also lead to switching among prey types as a result of changes in the abundances of other actors in the food web. Specifically, the marginal increase in mortality $\left(\mu_{m}\right)$ would depend on the abundance of top predators present, and thus factor into the grazers' decision as to which feeding mode to adopt. Such behaviourally (and trait-) mediated indirect interactions are apparently active in marine ecosystems (e.g. Kaartvedt et al. 2005), and are thought to be important shapers of food-web architecture and dynamics (Schmitz et al. 2008).

\section{CONCLUSIONS}

It is becoming apparent that the characteristics of the marine pelagic ecosystem predicted by models are critically dependent on the form of functional response curves used to describe the impact of grazrs on a diverse prey community (Mitra \& Flynn 2006, Mitra et al. 2007). There is a clear and immediate need to place these descriptor functions on a mechanistically credible and theoretically sound foundation. In this, we follow Smith et al. (2011) in espousing optimality in terms of fitness as a powerful conceptual tool in model development.

We propose a general algorithm for estimating optimal foraging mode on a mixed prey diet composed of 2 steps. Firstly, for each feeding mode (e.g. cruise, scanning current, cruise), possible ingestion rates are determined by prey profitability (from most profitable to some critical cut-off), the abundance of prey types, and their encounter kernel (a function of feeding mode and prey motility for instance). Secondly, the actual feeding mode (and thus actual ingestion rate, dietary inclusion and grazing pressure per prey class) is determined by the feeding mode that maximises fitness. This can be expressed either as a single optimal mode, or a weighted ensemble based on relative fitness afforded different feeding modes.

Implementing these algorithms will make ecosystem models more consistent 
in applying sound evolutionary principles and physics rather than arbitrary parameterisations of processes to stabilise models or maintain diversity.

Acknowledgements. This work was supported by the Research Council of Norway through a grant to Ø.F., and by the Danish Council for Strategic Research through a grant to A.W.V. We would like to thank Dag L. Aksnes, Andrew Barton, Sigrunn Eliassen, Mick Follows and Fi Prowe for fruitful discussions in preparing this manuscript.

\section{LITERATURE CITED}

Abrams PA (1984) Foraging time optimization and interactions in food webs. Am Nat 124:80-96

Abrams PA (2010) Implications of flexible foraging for interspecific interactions: lessons from simple models. Funct Ecol 24:7-17

Anderson TR, Gentleman WC, Sinha B (2010) Influence of grazing formulations on the emergent properties of a complex ecosystem model in a global ocean general circulation model. Prog Oceanogr 87:201-213

Banas NS (2011) Adding complex trophic interactions to a size-spectral plankton model: emergent diversity patterns and limits on predictability. Ecol Model 222:2663-2675

Charnov EL (1976) Optimal foraging: attack strategy of a mantid. Am Nat 110:141-151

Cohen JE, Pimm SL, Yodzis P, Saldana J (1993) Body sizes of animal predators and animal prey in food webs. J Anim Ecol 62:67-78

Dutz J, Koski M, Jónasdóttir SH (2008) Copepod reproduction is unaffected by diatom aldehydes or lipid composition. Limnol Oceanogr 53:225-235

Fasham MJR, Ducklow HW, Mckelvie SM (1990) A nitrogen-based model of plankton dynamics in the oceanic mixed layer. J Mar Res 48:591-639

Fiksen Ø, Jørgensen C (2011) Model of optimal behaviour in fish larvae predicts that food availability determines survival, but not growth. Mar Ecol Prog Ser 432:207-219

Follows MJ, Dutkiewicz S (2011) Modeling diverse communities of marine microbes. Annu Rev Mar Sci 3:427-451

Fuchs HL, Franks PJS (2010) Plankton community properties determined by nutrients and size-selective feeding. Mar Ecol Prog Ser 413:1-15

Gentleman WC, Neuheimer AB (2008) Functional responses and ecosystem dynamics: how clearance rates explain the influence of satiation, food-limitation and acclimation. J Plankton Res 30:1215-1231

Gentleman W, Leising A, Frost B, Strom S, Murray J (2003) Functional responses for zooplankton feeding on multiple resources: a review of assumptions and biological dynamics. Deep-Sea Res II 50:2847-2875

Gilliam JF, Fraser DF (1987) Habitat selection under predation hazard: test of a model with foraging minnows. Ecology 68:1856-1862

Gismervik I, Andersen T (1997) Prey switching by Acartia clausi: experimental evidence and implications of intraguild predation assessed by a model. Mar Ecol Prog Ser 157:247-259

Hansen PJ, Bjornsen PK, Hansen BW (1997) Zooplankton grazing and growth: scaling within the $2-2,000-\mu \mathrm{m}$ body size range. Limnol Oceanogr 42:687-704

Heckmann L, Drossel B, Brose U, Guill C (2012) Interactive effects of body-size structure and adaptive foraging on food-web stability. Ecol Lett 15:243-250

Holling CS (1959) Some characteristics of simple types of

Editorial responsibility: Jake Rice,

Ottawa, Canada predation and parasitism. Can Entomol 91:385-398

Kaartvedt S, Rostad A, Fiksen Ø, Melle W, Torgersen T, Breien MT, Klevjer TA (2005) Piscivorous fish patrol krill swarms. Mar Ecol Prog Ser 299:1-5

Kiørboe T (2011) How zooplankton feed: mechanisms, traits and trade-offs. Biol Rev Camb Philos Soc 86:311-339

Kiørboe T, Saiz E, Viitasalo M (1996) Prey switching behaviour in the planktonic copepod Acartia tonsa. Mar Ecol Prog Ser 143:65-75

Krebs CJ, Kacelnik A (1991) Decision making. In: Krebs CJ, Davies NB (eds) Behavioural ecology: an evolutionary approach. Blackwell Scientific Publications, Oxford, p 105-136

Mangel M, Clark CW (1988) Dynamic modeling in behavioral ecology. Princeton University Press, Princeton, NJ

> Mariani P, Visser AW (2010) Optimization and emergence in marine ecosystem models. Prog Oceanogr 84:89-92

> Mitra A, Flynn KJ (2006) Accounting for variation in prey selectivity by zooplankton. Ecol Model 199:82-92

Mitra A, Flynn KJ, Fasham MJR (2007) Accounting for grazing dynamics in nitrogen-phytoplankton-zooplankton models. Limnol Oceanogr 52:649-661

Murdoch WW, Avery S, Smyth MEB (1975) Switching in predatory fish. Ecology 56:1094-1105

Paffenhöfer GA (1984) Does Paracalanus feed with a leaky sieve? Limnol Oceanogr 29:155-160

Petchey OL, Beckerman AP, Riede JO, Warren PH (2008) Size, foraging, and food web structure. Proc Natl Acad Sci USA 105:4191-4196

> Prowe AEF, Pahlow M, Dutkiewicz S, Follows M, Oschlies A (2012a) Top-down control of marine phytoplankton diversity in a global ecosystem model. Prog Oceanogr 101:1-13

Prowe AEF, Pahlow M, Oschlies A (2012b) Controls on the diversity-productivity relationship in a marine ecosystem model. Ecol Model 225:167-176

Real LA (1977) Kinetics of functional response. Am Nat 111: 289-300

Schmitz OJ (2010) Resolving ecosystem complexity. Princeton University Press, Princeton, NJ

Schmitz OJ, Grabowski JH, Peckarsky BL, Preisser EL, Trussell GC, Vonesh JR (2008) From individuals to ecosystem function: toward an integration of evolutionary and ecosystem ecology. Ecology 89:2436-2445

Sheldon RW, Prakash A, Sutcliffe WH Jr (1972) The size distribution of particles in the ocean. Limnol Oceanogr 17: 327-340

Sjöberg S (1980) Zooplankton feeding and queuing theory. Ecol Model 10:215-225

Smith SL, Pahlow M, Merico A, Wirtz KW (2011) Optimalitybased modeling of planktonic organisms. Limnol Oceanogr 56:2080-2094

Stock CA, Powell TM, Levin SA (2008) Bottom-up and topdown forcing in a simple size-structured plankton dynamics model. J Mar Syst 74:134-152

Visser AW (2007) Motility of zooplankton: fitness, foraging and predation. J Plankton Res 29:447-461

Visser AW, Mariani P, Pigolotti S (2009) Swimming in turbulence: zooplankton fitness in terms of foraging efficiency and predation risk. J Plankton Res 31:121-133

Williamson CE (1980) The predatory behavior of Mesocyclops edax: predator preferences, prey defenses, and starvationinduced changes. Limnol Oceanogr 25:903-909

Wirtz KW (2012a) Intermittency in processing explains the diversity and shape of functional grazing responses. Oecologia 169:879-894

Wirtz KW (2012b) Who is eating whom? Morphology and feeding type determine the size relation between planktonic predators and their ideal prey. Mar Ecol Prog Ser 445:1-12

Submitted: June 11, 2012; Accepted: July 27, 2012

Proofs received from author(s): January 7, 2013 